\title{
Eixos Hormonais na Obesidade: Causa ou Efeito?
}

revisão

\author{
RoBerta A. LORDELO \\ MARCIO C. MANCINI \\ Cíntia Cercato \\ ALFREDO HALPERN
}

\section{Grupo de Obesidade e Síndrome Metabólica do Serviço de Endocrinologia $e$ Metabologia do Hospital das Clínicas da Faculdade de Medicina da USP, São Paulo, SP.}

Diversas alterações endócrinas são descritas na obesidade. $\mathrm{O}$ eixo corticotrófico encontra-se hiper-responsivo, com maior depuração dos hormônios e nível de cortisol normal. A caracterização do pseudo-Cushing é importante. A leptina parece ser um hormônio permissivo para o desencadeamento da puberdade. Em adultos, as gonadotrofinas são normais, hiperandrogenismo e hiperestrogenismo são encontrados. Nas mulheres, a resistência insulínica é central no desenvolvimento da síndrome dos ovários policísticos (SOP), associada a hiperandrogenemia ovariana. Nos obesos, GH geralmente é baixo e IGF1 normal. A função tireoidiana é habitualmente normal nos obesos. (Arq Bras Endocrinol Metab 2007;51/1:34-41)

Descritores: Obesidade; Eixos endócrinos; Hormônios; ACTH; TSH; LH; FSH; Estrogéno; Testosterona; Leptina; $\mathrm{GH}$

\begin{abstract}
Hormonal Axes in Obesity: Cause or Effect?

Several endocrine changes have been described in the obesity state. The corticotropic axis is hyperresponsive and there is enhancement of hormonal clearance, but cortisol levels are within the normal range. It is important to characterize a pseudo-Cushing in obesity. Leptin seems to be a permissive hormone for the beginning of puberty. In adults, gonadotropines are normal, and hyperandrogenism and hyperestrogenism are found. In women, insulin resistance has a central role in polycystic ovarian syndrome (POS), which is associated to ovarian hyperandrogenemia. In obese subjects, growth hormone $(\mathrm{GH})$ is generally low and IGF1 is normal. Thyroid function is commonly normal in obese subjects.
\end{abstract} (Arq Bras Endocrinol Metab 2007;51/1:34-41)

Keywords: Obesity; Endocrine axis; Hormones; ACTH; TSH; LH; FSH; Estrogen; Testosterone; Leptin; GH

$\mathrm{O}$ NÍVEL E A FUNÇÃO DE DIVERSOS hormônios encontram-se alterados na obesidade. Não é conhecido se as modificações são meramente adaptativas e fisiológicas ou se possuem algum papel na gênese ou perpetuação da obesidade. Em estudos experimentais nos quais as alterações foram restauradas para o nível "normal", não foram observados maior perda de peso ou melhor prognóstico da obesidade. A função endócrina do tecido adiposo pode ter papel etiológico, pois as alterações variam com a gravidade da obesidade e o fenótipo de distribuição de gordura corporal. $\mathrm{O}$ conhecimento do perfil hormonal peculiar do paciente obeso é importante para: 1) compreender a fisiopatologia das complicações associadas ao excesso de peso; 2 ) evitar erros diagnósticos (por exemplo, atribuição das alterações encontradas a processos orgânicos); 3 ) motivar estudos que possam resultar em intervenções terapêuticas.
Recebido em 21/04/06 Aceito em 30/06/06 


\section{EIXO HIPOTÁLAMO-HIPÓFISE-ADRENAL}

O eixo hipotálamo-hipófise-adrenal apresenta-se hiper-responsivo nos indivíduos obesos. Observa-se resposta aumentada do cortisol a estímulo com ACTH, CRH e refeição mista (1). Há maior freqüência na liberação dos pulsos de ACTH, com menor amplitude de pulso, mas o nível basal é normal.

Existem diversas evidências na literatura de que há uma maior ativação do eixo HHA em pacientes obesos, particularmente naqueles com distribuição central de gordura. Pasquali e cols., em 1993, demonstraram que mulheres com obesidade central apresentaram um aumento significativo de cortisol e ACTH após estímulo com CRH em relação a controles não obesas, ou mulheres com obesidade periférica (2). Há maior freqüência na liberação dos pulsos de $\mathrm{ACTH}$, com menor amplitude de pulso, mas o nível basal é normal. Não é conhecido se esta alteração influencia a liberação de cortisol pelas glândulas adrenais.

Alternativamente, uma anormalidade no metabolismo dos glicocorticóides, levando ao maior clearance metabólico de cortisol, poderia estimular o eixo HHA para compensar as alterações periféricas. O clearance metabólico de cortisol encontra-se elevado em obesos. A diminuição da globulina ligadora de corticoesteróides e a alta densidade de receptores de glicocorticóides (GC) periféricos são alterações que favorecem a metabolização do cortisol (3). Os adipócitos viscerais possuem maior densidade de receptores de GC que os adipócitos do tecido adiposo periférico (4). Estudo sobre a farmacocinética de cortisol na obesidade, realizado por Lottenberg e cols., em 1998, demonstrou que o clearance metabólico de cortisol apresentava forte correlação com a quantidade de gordura abdominal. Portanto, quanto maior a quantidade de gordura visceral, maior o clearance metabólico de cortisol, resultando em redução dos seus níveis plasmáticos, e maior estímulo do eixo HHA (5).

Assim, o aumento do clearance de cortisol é compensado por um aumento da produção (6), ou esta pode decorrer primariamente da hiperativação hipotálamo-hipofisária. Conseqüentemente, o cortisol sérico basal é normal (figura 1).

Uma forma de se avaliar clearance metabólico de cortisol é através da mensuração da excreção de cortisol urinário nas 24 horas. O cortisol urinário livre pode ser normal ou levemente aumentado, mas raramente maior do que quatro vezes o valor de referência (7). No nosso serviço, encontramos que $9,7 \%$ dos obesos apresentavam níveis elevados de cortisol urinário (8).

A resposta à supressão com doses baixas de dexametasona $(0,5-1,0 \mathrm{mg})$ pode ser reduzida, mas a su-

\section{Obesidade andróide}

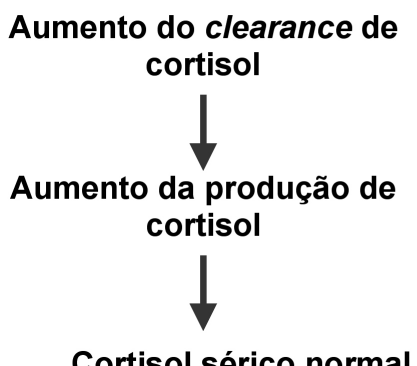

- Densidade dos receptores periféricos

- Diminuição da CBG

- Hiperativação do eixo a estímulos

- Hiperresponsividade ao

ACTH

\section{Cortisol sérico normal}

Figura 1. Alterações do cortisol na obesidade.

pressão é normal com doses mais altas (9) ou no teste do CRH mais dexametasona (6). O ritmo circadiano é normal, apesar de alguns autores terem documentado nível menor de cortisol sérico pela manhã em obesos, quando comparados com indivíduos de peso normal (10).

Em resumo, apesar das sutis alterações encontradas, não há evidência de hipercortisolismo clínico (bioquímico) em indivíduos obesos. Em revisão recente, Salehi e cols. (11) afirmam que os dois parâmetros principais utilizados para avaliar hipercortisolismo sistêmico são o cortisol urinário livre e o cortisol sérico, sendo o último mais importante e preciso. Salientam que a interpretação apropriada do cortisol urinário requer correção para a massa metabolicamente ativa, melhor realizada expressando o resultado por grama de creatinina urinária. Lembram, também, que a avaliação do cortisol sérico pode ser influenciada por flutuações momentâneas devido a secreção episódica. Uma avaliação mais apropriada seria feita avaliando a concentração média nas 24 horas. Os autores concluem que o cortisol urinário livre corrigido é normal em indivíduos obesos e que a concentração média de cortisol em 24 horas é levemente subnormal.

Apesar de não haver hipercortisolismo bioquímico, o hipercortisolismo funcional presente na obesidade abdominal poderia contribuir para a síndrome metabólica, cujas características se assemelham em muito às manifestações da síndrome de Cushing.

Existe aumento da atividade glicocorticóide na gordura abdominal devido a maior atividade local da enzima 11-beta-hidroxiesteróide desidrogenase do tipo $1(11 \beta H S D-1)$, que catalisa a interconversão de cortisona para cortisol na célula-alvo.

Estudos com ratos Zucker obesos, resistentes à leptina, demonstraram aumento da atividade da 11ßHSD-1 no tecido adiposo omental, acarretando uma maior geração de glicocorticóide local, e conseqüentemente maior ativação de seu receptor, promovendo obesidade (12). 
Um dos estudos de maior relevância sobre a participação da $11 \beta$ HSD-1 na obesidade foi o de Masuzaki e cols., em 2001 (13). Neste estudo, os autores desenvolveram um modelo transgênico de ratos com superexpressão de $11 \beta$ HSD-1 exclusivamente no tecido adiposo. Os animais transgênicos apresentavam níveis séricos de corticosterona (equivale ao cortisol em humanos) semelhantes aos animais não transgênicos, porém os níveis de corticosterona no tecido adiposo eram significativamente aumentados. Os ratos com hiperexpressão da $11 \beta$ HSD-1 apresentaram maior ganho de peso, principalmente na região abdominal, especialmente após dieta rica em gordura. $\mathrm{O}$ aumento de peso foi paralelo ao aumento do consumo energético, indicando que o aumento da atividade da 11ßHSD-1 no tecido adiposo pode levar a hiperfagia. Os animais transgênicos eram marcadamente hiperglicêmicos e hiperinsulinêmicos e apresentavam níveis plasmáticos aumentados de ácidos graxos livres, triglicérides e leptina. Através deste estudo, Masuzaki e cols. indicaram que o aumento da atividade da $11 \beta$ HSD-1 no tecido adiposo foi capaz de causar hiperfagia, obesidade visceral e síndrome metabólica.

Estudos recentes têm avaliado a atividade da 113HSD-1 no tecido adiposo em humanos. Rask e cols., em 2001, avaliaram homens saudáveis que foram submetidos a biópsia de tecido adiposo subcutâneo abdominal, e verificaram que quanto maior o IMC, maior a atividade da 11ßHSD-1 (14). Outro grupo demonstrou um aumento da expressão do gene da $11 \beta$ HSD-1 em biópsia de tecido adiposo de mulheres, e que houve uma correlação positiva entre expressão e anormalidades metabólicas (15).

\section{EIXO-HIPOTÁLAMO-HIPÓFISE-GONADAL}

\section{Puberdade}

A observação de que crianças desnutridas não entram em puberdade e que crianças obesas iniciam a puberdade em idade mais precoce é antiga. Estudos epidemiológicos corroboraram a observação clínica da importância do tecido adiposo no desencadeamento e manutenção da atividade reprodutiva (16).

A possibilidade de um elo etiológico foi observada inicialmente em camundongos. Em 1996, foi notado que camundongos $o b / o b$ (que não produzem leptina) não entravam em puberdade e que a injeção de leptina recombinante desencadeava puberdade (17).

Administração de leptina a camundongos $o b / o b$ leva a aumento do nível basal de LH. Em camundongos selvagens, o jejum de 48 horas acarreta redução da freqüência de pulsos de LH e, paralelamente, a leptina encontra-se suprimida. A administração exógena de leptina evita a redução da freqüência de pulso de LH. A ação central da leptina sobre as gonadotrofinas foi evidenciada através da injeção intracerebroventricular de anticorpo antileptina em camundongos, o que causou redução na secreção pulsátil de LH (18).

A leptina pode ter uma ação direta na regulação de GnRH hipotalâmico, sendo evidências a favor a presença de receptores no hipotálamo e a capacidade da leptina de estimular liberação de GnRH em extratos hipotalâmicos in vitro.

As concentrações de leptina oscilam durante o desenvolvimento puberal em ratos, primatas e humanos. Em meninas, a concentração aumenta progressivamente durante o desenvolvimento puberal. Em meninos, a leptina aumenta antes da puberdade e durante as fases iniciais, e depois declina progressivamente (19). Estas diferenças podem contribuir para a distribuição regional de gordura característica de cada sexo (20).

A leptina em humanos parece ter um papel permissivo para o início da puberdade, sendo um fator necessário mas não suficiente para o seu desencadeamento.

\section{Esteróides sexuais e gonadotrofinas no adulto}

Serão descritas as peculiaridades do metabolismo estrogênico e androgênico no obeso adulto em ambos os sexos. As gonadotrofinas, de modo geral, são normais ou apresentam alterações discretas. Anovulação crônica e infertilidade são associadas à obesidade. A síndrome dos ovários policísticos (SOP) é responsável pela maior parte dos casos de infertilidade.

\section{Gonadotrofinas}

Geralmente, a obesidade não está associada a alterações nos níveis de gonadotrofinas. Não existe alteração de resposta à administração de GnRH ou clomifeno (21). Diferenças na pulsatilidade de LH não foram encontradas em obesas antes da menopausa (22).

Homens com obesidade grave têm níveis de testosterona livre baixos sem concomitante aumento de LH, sugerindo um estado de hipogonadismo hipogonadotrófico. As alterações são reversíveis com perda ponderal (23).

Em algumas mulheres obesas após a menopausa, observam-se níveis de gonadotrofinas menores do que o esperado na condição de falta de retroalimentação negativa estrogênica (24).

Como será descrito a seguir, mulheres obesas com SOP apresentam alterações de gonadotrofinas características da condição. 


\section{Esteróides sexuais}

Na obesidade, a produção androgênica adrenal e ovariana encontra-se elevada, apresentando correlação positiva com IMC. Nas mulheres, a obesidade abdominal é fortemente associada a hiperandrogenemia. Nos homens, entretanto, a produção androgênica testicular é reduzida, apresentando correlação inversa com o grau de obesidade abdominal.

Assim como acontece com o cortisol, o aumento da produção adrenal pode ser uma compensação para um clearance aumentado. Os 17-cetoesteróides urinários são freqüentemente elevados em indivíduos obesos. Também parece haver hiper-responsividade dos andrógenos adrenais ao estímulo corticotrófico, como demonstrado por aumento de deidroepiandrosterona (DHEA) e da relação DHEA/17-OH progesterona após administração exógena de ACTH. Este achado também apresenta correlação positiva com IMC (25).

A globulina ligadora de hormônios sexuais (SHBG) encontra-se diminuída em indivíduos obesos causando grande impacto no metabolismo e na ação dos esteróides sexuais. Esta alteração pode também facilitar o aumento do clearance androgênico. A diminuição da SHBG decorre de menor produção hepática e correlaciona-se inversamente com IMC, hiperinsulinemia e relação cintura-quadril (26). Há maior quantidade de testosterona livre (bioativa) nas mulheres. Em homens moderadamente obesos, a testosterona total é baixa devido à redução de SHBG, mas a testosterona livre é normal. Como já mencionado, a testosterona livre poder ser reduzida em obesos graves (IMC>40) devido à redução de freqüência e amplitude de pulso de LH nesses indivíduos (27).

A obesidade é uma condição de hiperestrogenismo, associada a aumento de risco para hiperplasia endometrial e carcinoma hormônio-sensível em mulheres. A aromatização, reação enzimática responsável pela conversão de andrógenos a estrógenos, ocorre no tecido adiposo de homens e mulheres (tanto na pré- como na pósmenopausa) e é fortemente relacionada ao peso corporal. A androstenediona é o principal substrato para formação periférica de estrógenos e é convertida a estrona. Disto resulta aumento da relação estrona/estradiol. A estrona também pode ser convertida em estradiol no próprio tecido adiposo por atividade $17 \beta$-hidróxi-esteróide-desidrogenase. Os níveis de estrógenos totais são iguais aos de mulheres magras, mas a fração livre encontra-se aumentada (28). Níveis de estrógenos também são elevados em homens obesos, mas não ocorrem sinais de feminilização.

O nível baixo de SHBG amplifica mais a ação estrogênica do que a androgênica. Isto decorre da menor afinidade da SHBG pelo estradiol e pela maior biotividade molar do estradiol $(\mathrm{pg} / \mathrm{ml})$ em relação à testosterona $(\mathrm{ng} / \mathrm{ml})$. Em mulheres após a menopausa, os níveis de estrona e estradiol apresentam correlação com o grau de obesidade (29).

O tecido adiposo é um grande reservatório de esteróides, já que eles concentram-se preferencialmente nos adipócitos do que no plasma (a concentração de esteróides na gordura é $2-13$ vezes maior do que no plasma), e o volume de gordura no obeso é muito maior que o espaço intravascular.

\section{Ovários e SOP}

A condição de hiperestrogenisno e hiperandrogenismo é comum em mulheres obesas. Entretanto, cerca de $50 \%$ das obesas destacam-se por terem um excesso de produção ovariana (com alterações morfológicas associadas) e alteração de gonadotrofinas que caracterizam a síndrome dos ovários policísticos.

No consenso de Roterdã (30), SOP é definida pela presença de pelo menos dois dos seguintes critérios: disfunção ovulatória (oligomenorréia, amenorréia, hemorragia uterina disfuncional, menstruação regular), hiperandrogenismo ou hiperandrogenemia e ovários policísticos (pelo menos um ovário com 12 ou mais folículos medindo 2-9 $\mathrm{mm}$ de diâmetro e/ou aumento de volume $>10 \mathrm{ml}$ ), excluídas causas secundárias.

Aumento absoluto do nível LH é encontrado em $60 \%$ e da relação $\mathrm{LH} / \mathrm{FSH}$ em $95 \%$ das pacientes (30). Este achado decorre de maior número de pulsos de GnRH por alteração hipotalâmica primária ou falta de inibição pela progesterona (anovulação).

Hiperandrogenemia, hiperinsulinemia e obesidade abdominal são condições independentemente relacionadas à SOP e cada uma capaz de perpetuar as demais condições.

A hiperinsulinemia parece ter um papel central na etiopatogenia da SOP. É capaz de elevar a androgenemia por estimular esteroidogênese ovariana e adrenal e reduzir SHBG (aumentando a biodisponibilidade). O estímulo à esteroidogênese ovariana ocorre diretamente através da ação em seu próprio receptor ou por ação cruzada no receptor de IGFl, ou até mesmo indiretamente por aumentar a biodisponibilidade de IGFl (31).

As evidências são reforçadas por estudos clínicos nos quais tratamento da hiperinsulinemia com diazóxido, metformina ou troglitazona resultaram em diminuição de andrógenos, aumento de SHBG e diminuição da atividade 17,20 liase (32).

A insulina altera a foliculogênese ovariana contribuindo para as alterações morfológicas e pode estimular diretamente a liberação de LH pela hipófise (22).

A leptina também pode ter um efeito modulatório na pulsatilidade do GnRH. O nível mais eleva- 
do de leptina em mulheres obesas com SOP está inversamente correlacionado com o nível basal de LH (33).

Uma perda de peso modesta $(2-7 \%)$ com modificações de estilo de vida promove redução do nível de andrógenos e melhora função ovulatória. Vários estudos com metformina 1,5 a $2 \mathrm{~g} /$ dia demonstram diminuição da insulinemia, aumento da sensibilidade a insulina e da SHBG, redução do nível de andrógenos e LH $(34,44)$. Os efeitos ocorrem mesmo em obesos que não perderam peso e em magros (23). Metformina regulariza ciclos menstruais e ovulação em 78-96\% dos pacientes (36).

\section{EIXO SOMATOTRÓFICO}

Nível reduzido de GH e baixa resposta de GH a estímulos são características da obesidade. A resposta a estímulo com clonidina, arginina, GHRH, GHRP6, hipoglicemia e exercício é reduzida $(37,38)$. Em modelos animais de obesidade genética, o número de células somatotróficas é reduzido na hipófise. Em humanos, constata-se diminuição do número de pulsos de GH e maior depuração. Todas estas alterações são revertidas em obesos que perdem peso.

Apesar do prejuízo na liberação de $\mathrm{GH}$, não há déficit absoluto na produção pois a administração combinada de GHRH e GHRP6 ou associação de propranolol aos estímulos normaliza a resposta do GH (39).

A leptina pode ser um dos fatores hipotalâmicos implicados nas alterações. Em animais, tem efeito inibitório na secreção de $\mathrm{GH}$ através de efeitos no GHRH e neuropeptídeo Y no hipotálamo (40). Elevações agudas ou crônicas nos níveis de ácidos graxos livres (AGL) ocasionam inibição da liberação de $\mathrm{GH}$ pela hipófise. AGL encontram-se elevados em condições de hiperinsulinemia tal como a obesidade (41).

Não parece haver déficit funcional de GH nos obesos, já que o nível de IGFl é normal. Há evidências de maior sensibilidade da ação do $\mathrm{GH}$, tais como maior nível de GHBPs (e conseqüentemente de receptores de $\mathrm{GH}$ ) e o achado de que administração de baixas doses de rhGH tem efeito estimulatório na produção de IGFI maior em obesos do que em indivíduos de peso normal (42). Além disso, a redução do peso após cirurgia bariátrica acarreta elevação marcante de parâmetros do perfil de secreção de 24 horas de GH (ritmo) em obesas grau III sem alteração do nível de ghrelina, sugerindo um papel limitado desta na regulação fisiológica do eixo somatotrófico (43).

Alguns acreditam que alterações periféricas nos níveis de IGFl e IGFBPl sejam as alterações primárias, levando secundariamente a redução da secreção de $\mathrm{GH}$. É conhecido que a hiperinsulinemia, característica da obesidade, induz maior produção de IGFl e menor produção de IGFBPl pelo fígado. IGFBPl é uma proteína ligadora de IGFl que inibe a sua atividade. Destas alterações induzidas pela insulina, resultaria maior quantidade de IGFI livre capaz de exercer feedback negativo na hipófise para secreção de GH (44) (figura 2).

É importante lembrar que crianças obesas têm crescimento linear normal e até um pouco acelerado em relação a crianças de peso normal, atingido altura final comparável. Deve-se estar atento à interpretação de testes de estímulo ao GH em crianças obesas em investigação para baixa estatura, pois a falta de resposta ao estímulo perde especificidade para o diagnóstico de déficit de GH.

Obesos com síndrome de apnéia obstrutiva do sono (SAOS) podem ter alterações mais profundas no eixo somatotrófico, com um verdadeiro déficit funcional. Em recente estudo, obesos com SAOS tiveram maior redução de resposta do $\mathrm{GH}$ em testes provocativos, níveis mais reduzidos de IGFl sem melhora após administração de rhGH em doses baixas (45). Os autores postulam que SAOS possa prejudicar o eixo somatotrófico independentemente da adiposidade. A hipóxia pode ser o fator mediador, uma vez que hipóxia aguda ou crônica reduz a síntese e liberação de $\mathrm{GH}$

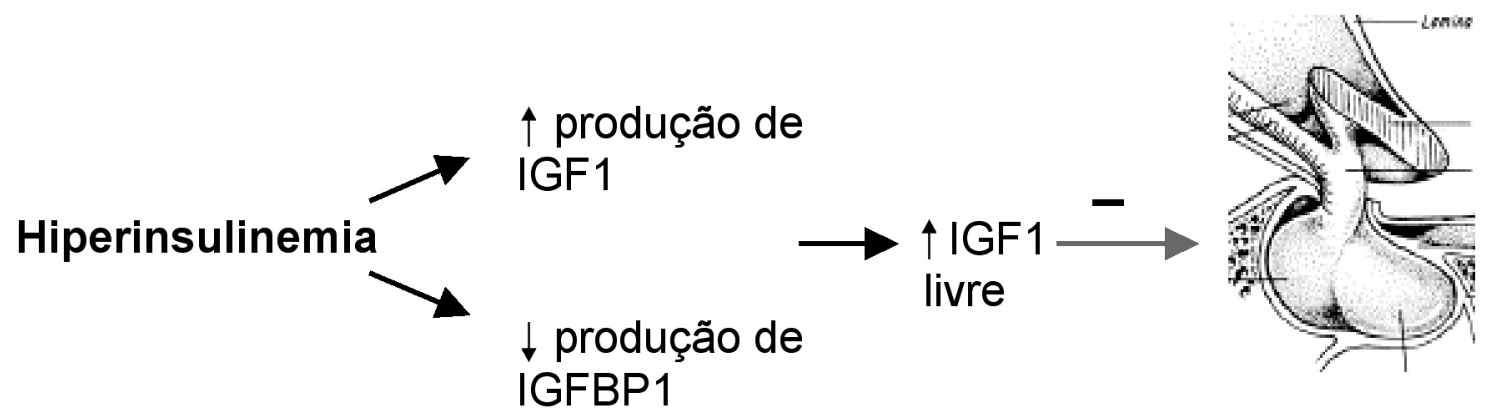

Figura 2. Hipótese para redução de GH na obesidade: mecanismo periférico. 
em animais e reduz a expressão do mRNA do IGFl por células endoteliais in vitro.

Alguns autores acreditam que as alterações do eixo somatotrófico de obesos possam ser mal-adaptativas. As características de distribuição de gordura corporal e dislipidemia similares aos pacientes com déficit de GH decorreriam do nível baixo de IGFl total.

A baixa concentração de $\mathrm{GH}$ na obesidade visceral levou à administração deste hormônio em indivíduos obesos. Devido ao seu efeito lipolítico seria esperada perda de peso, e devido ao seu efeito anabólico protéico seria esperada uma proteção contra o balanço nitrogenado negativo freqüentemente acompanhado nas dietas hipocalóricas. Além disso, o GH poderia estar associado a uma redução de gordura visceral com melhora dos efeitos metabólicos desta distribuição.

O racional para estas expectativas é suportado por estudos de reposição de $\mathrm{GH}$ em pacientes com deficiência primária de GH que apresentaram aumento de massa muscular e redistribuição da gordura intraabdominal para depósitos periféricos após a administração crônica do hormônio.

Realizamos, no Grupo de Obesidade e Síndrome Metabólica do Hospital das Clínicas da Faculdade de Medicina da Universidade de São Paulo, um estudo prospectivo, randomizado, duplo-cego, em 40 homens não diabéticos de 20 a 50 anos com distribuição visceral de gordura, tratados com GH $(0,050 \mathrm{U} / \mathrm{kg} / \mathrm{dia})$ ou placebo por três meses. O objetivo deste estudo foi avaliar os efeitos do GH sobre composição corporal e fatores de risco cardiovascular na obesidade visceral. Observou-se redução de peso $(3,5 \pm 2,9 \mathrm{~kg}), \operatorname{IMC}(1,2$ $\left.\pm 1,0 \mathrm{Kg} / \mathrm{m}^{2}\right)$, relação cintura/quadril $(0,04 \pm 0,01 \mathrm{~cm})$ e massa adiposa $(2,4 \pm 1,0 \mathrm{Kg})$, bem como colesterol total $(4,0 \pm 3,3 \mathrm{mg} / \mathrm{dL})$ e LDL-colesterol $(5,7 \pm 2,7$ $\mathrm{mg} / \mathrm{dL}$ ) no grupo $\mathrm{GH}$, com variações percentuais significantes em relação ao placebo. Apesar destes achados em nosso grupo, os benefícios e riscos do uso de $\mathrm{GH}$ em pacientes obesos a longo prazo ainda são desconhecidos.

Em uma meta-análise de 16 estudos publicados sobre a administração de $\mathrm{GH}$ em obesos com distribuição predominantemente central de gordura, não se encontraram evidências para benefícios metabólicos da administração de GH na obesidade na ausência de deficiência de GH verdadeira. Pelo contrário, quase todos os estudos relataram efeitos negativos do GH no metabolismo de glicose. Portanto, apesar da perda de gordura visceral ser atingida com a administração de $\mathrm{GH}$, este benefício é perdido pela piora da resistência insulínica induzida pelo GH. Além disso, a administração de GH foi acompanhada de outros efeitos colaterais, como retenção hídrica, artralgias e síndrome do túnel do carpo (46).
Portanto, apesar de os níveis de GH serem reduzidos na obesidade, particularmente do tipo visceral, a perda de peso pode restaurar a secreção normal deste hormônio. O uso exógeno de GH com objetivo de perda de peso não é recomendado, uma vez que sua eficácia e segurança a longo prazo ainda não foram comprovadas (47). Indicações alternativas para uso de GH que não o déficit documentado não são consensuais na literatura.

\section{EIXO TIREOTRÓFICO}

Frente ao importante papel dos hormônios tireoidianos na regulação da termogênese, a dinâmica do eixo hipotálamo-hipófise-tireoidiano na obesidade já foi amplamente estudada.

Em obesos em estado neutro de balanço energético, os níveis de TSH, T4 e T3 são normais. As alterações clássicas descritas são as encontradas no estado de jejum (aumento de RT3 e redução de T3) e no estado de hiperalimentação (aumento de T3 e redução de RT3), encaradas como respostas que tentam manter o peso corporal.

Quando avalia-se a função tireoidiana no indivíduo obeso, deve-se considerar os efeitos do balanço energético atual, caso esteja submetido a dieta hipocalória ou em plena hiperalimentação.

Vários estudos foram conduzidos com administração de hormônios tireoidianos a obesos em dieta restrita, na tentativa de reverter as alterações metabólicas que conduzem à redução da taxa metabólica basal (TMB). Diversos estudos utilizaram doses elevadas de T3, obtendo-se aumento do nível sérico de T3 e correlação negativa com o peso final (48).

A reposição de T3 a obesos com restrição calórica moderada ocasiona aumento da TMB e maior perda de peso, mas somente em doses altas associadas a sérios efeitos adversos cardiovasculares e excessivo consumo de massa muscular (49).

Um estudo sugere que a redução de T3 associada a dietas restritas pode ser parcialmente prevenida pela suplementação de zinco e selênio devido à habilidade desses minerais em aumentar a atividade das deiodinases I e II (67\% e 47\%, respectivamente) (50).

Recentemente, estudos têm sido conduzidos com administração de doses baixas de T3 (5-20 $\mu \mathrm{g})$ a obesos em dieta restrita com o objetivo de prevenir hipoglicemia, dislipidemia e aumentar SHBG (51).

Pacientes com hipotireoidismo pesam, em média, $15 \%$ a $30 \%$ mais que indivíduos eutireoidianos. Mesmo após terapia adequada, cerca de 7\% ainda permanece acima do peso. Em estudo transversal, foi encontrada uma 
associação discreta entre hipotireoidismo subclínico e IMC em mulheres, mas uma associação em direção oposta em homens. Hipotireoidismo clínico ou subclínico parece não ser mais comum em obesos do que indivíduos de peso normal (52). Apesar disto, numa casuística de 72 indivíduos com obesidade mórbida com indicação cirúrgica de derivação gastrojejunal, a incidência de hipotireoidismo subclínico foi de $25 \%$, tendo o TSH normalizado um ano após a cirurgia em todos os indivíduos (53).

Pacientes hipotireóideos em reposição com levotiroxina apresentam variações na TMB quando apresentam-se em eutireoidismo, hipertireoidismo leve ou hipotireoidismo (54). As implicações destas variações da TMB no IMC não estão definidas. Estudos nos quais T4 foi administrado a pacientes com hipotireoidismo leve não mostraram mudança significativa de IMC em seguimento de até 6 meses (55). A leptina influencia a regulação central da função tireoidiana, através do estímulo hipotalâmico do TRH. No hipotireoidismo, os níveis de leptina são elevados, talvez como forma de contrapor ao acúmulo de lipídeos e energia na ausência de T3 (56).

\section{REFERÊNCIAS}

1. Rosmond R, Dallman MF, Björntorp P. Stress-related cortisol secretion in men: relationship with abdominal obesity and endocrine, metabolic and hemodynamic abnormalities. J Clin Endocrinol Metab 1998;83(6):1853-9.

2. Pasquali R, Cantobelli S, Casimirri F, Capelli M, Bortoluzzi L, Flamia $R$ et al. The hypothalamic-pituitary-adrenal axis in obese women with different patterns of body fat distribution. J Clin Endocrinol Metab 1993;77(2):341-6.

3. Rebuffe-Scrive M, Bronnegard M, Nilsso A, Eldh J, Gustafsson JA, Bjorntorp P. Steroid hormone receptors in human adipose tissues. J Clin Endocrinol Metab 1990;71:1215-9.

4. Bronnegard M, Arner P, Hellstrom Let, Akner G, Gustafsson JA. Glucocorticoid receptor messenger ribonucleic acid in different regions of human adipose tissue. Endocrinology 1990;127:1689-96.

5. Lottenberg SA, Giannella-Neto D, Derendorf H, Rocha M, Bosco A, Carvalho SV, et al. Effect of fat distribution on the pharmacokinetics of cortisol in obesity. Int J Clin Pharmacol Ther 1998;36(9):501-5.

6. Tales A, Graves L, Burke CW, Fotherby K, Fraser R. Free cortisol in obesity: effect of fasting. Acta Endocrinologica 1976;81(2):321-9.

7. Beauregard C, Dickstein G, Lacroix A. Classic and recent etiologies of Cushing syndrome: diagnosis and therapy. Treat Endocrinol 2002;1(2):79-94.

8. Arguello AMC, Cercato C, Barroso RL, et al. Urinary free cortisol levels in obese patients. Int J Obes Relat Metab Disord 2002;26(suppl. 1):S198.

9. Streeten DHP, Stevenson GT, Dalakos TG. The diagnosis of hypercortisolism: Biochemical criteria differentiating patients form lean and obese normal subjects and from females on oral contraceptives. J Clin Endocrinol Metab 1969;29:1191.

10. Strain GW, Zumoff B, Strain JJ. Cortisol production in obesity. Metabolism 1980;29:980-5.

11. Salehi M, Ferenczi A, Zumoff B. Obesity and cortisol status. Horm Metabol Res 2005;37(4):193-7.

12. Livingstone DE, Kenyon CJ, Walker BR. Mechanisms of dysregulation of 11 beta-hydroxysteroid dehydrogenase type 1 in obese Zucker rats. J Endocrinol 2000;167(3):533-9.
13. Masuzaki H, Paterson J, Shinyama H, Morton NM, Mullins JJ, Seckl JR, et al. A transgenic model of visceral obesity and the metabolic syndrome. Science 2001;294(5549):2166-70.

14. Rask E, Olsson T, Soderberg S, Andrew R, Livingstone DE, Johnson $\mathrm{O}$, et al. Tissue-specific dysregulation of cortisol metabolism in human obesity. J Clin Endocrinol Metab 2001;86(3):1418-21.

15. Engeli S, Bohnke J, Feldpausch M, Gorzelniak K, Heintze U, Janke J, et al. Regulation of 11 beta-HSD genes in human adipose tissue: influence of central obesity and weight loss. Obes Res 2004;12(1):9-17.

16. Frish RE. Body fat, menarche, fitness and fertility. In: Frish RE (ed.). Adipose Tissue and Reproduction. Basel: S. Karger, 1990. pp. 1-26.

17. Chehab FF, Lim ME, Ronghue LL. Correction of the sterility defect in homozygous obese female mice by treatment with the human recombinant leptin. Nat Genet 1996:12:318-20.

18. Foster DL, Nagatani S. Physiological perspectives on leptin as a regulator of reproduction: role in timing puberty. Biol Reprod 1999;60:205-15.

19. Kiess W, Reich A, Meyer K, Glasow A, Deutscher J, Klammt J, et al. A role for leptin in sexual maturation and puberty? Horm Res 1999;51(S3):55-63.

20. Roemmich JN, Rogol AD. Role of leptin during childhood growth and development. Endocrinol Metab Clin North Am 1999;28(4):749-64.

21. Glass AR, Swerdloff RS, Brady GA, Dahms WT, Atkinson RL. Low serum testosterone and sex hormone binding globulin in massively obese men. J Clin Endocrinol Metab 1977;45:1211-9.

22. Kopelman PG, White N, Pilkington TRE, Jeffcoate SL. The effect of weight loss on steroid secretion and binding in massively obese women. Clin Endocrinol 1981;14:113-6.

23. Lima N, Cavaliere H, Knobel M, Halpern A, Medeiros-Neto G. Decreased androgen levels in massively obese men may be associated with impaired function of the gonadostat. Int $\mathbf{J}$ Obes Relat Metab Disord 2000;24(11):1433-7.

24. Malacara JM, Fajardo ME, Nava LE. Gonadotropins at menopause: the influence of obesity, insulin resistance, and estrogens. Steroids 2001;66(7):559-67.

25. Kopelman PG. Hormones and obesity. Baillière's Clin Endocrinol Metab 1994:8(3):549-75.

26. Evans DJ, Hoffman RG, Kalkhoff R, Kissebah AH. Relationship of androgenic activity to body fat topography, fat cell morphology and metabolic aberrations in premenopausal women. J Clin Endocrinol Metab1983;57:304-10.

27. Giagulli VA, Kaufman JM, Vermeulen A.Pathogenesis of decreased androgen levels in obese men. J Clin Endocrinol Metab 1994;79(4):997-1000.

28. Kopelman PG, Pilkington TRE, White N, Jeffcoate SL. Abnormal sex steroid secretion and binding in massively obese women. Clin Endocrinol 1980;12:363-9.

29. Meldrum DR, Davidson B J, Tataryn IV, Judd HL. Changes in circulating steroids with aging in post-menopausal women. Obstet Gynecol 1981;57:624-8.

30. The Rotterdam ESHRE/ASRM-sponsored PCOS consensus workshop group. Revised 2003 consensus on diagnostic criteria and long-term health risks related to polycystic ovary syndrome. Hum Reprod 2004;19(1):41-7.

31. Dunaif A. Insulin resistance and the Polycystic Ovary Syndrome: mechanism and implications for pathogenesis. Endocr Rev 1997;18(6):774-800.

32. Ehrmann DA. Polycystic Ovary Syndrome. N Engl J Med 2005:352:1223-36.

33. Spritzer PM, Poy M, Wiltgen D. Leptin concentrations in hirsute women with polycystic ovary syndrome or idiopathic hirsutism: influence on LH and relationship with hormonal, metabolic, and anthropometric measurements. Hum Rep 2001:16:1340-6.

34. Hoeger KM, Kochman L, Wixom N, Craig K, Miller RK, Guzick DS. A randomized, 48 week, placebo-controlled trial of intensive lifestyle modification and/or metformin therapy in overweight women with polycystic ovary syndrome: a pilot study. Fertil Steril 2004;82:421-9. 
35. Nestler JE, Jakubowicz DJ, Evans WS, Pasquali R. Effects of metformin on spontaneous and clomiphene-induced ovulation in the polycystic ovary syndrome. N Engl J Med 1998;338:1876-80.

36. Velazquez EM, Acosta A, Mendoza SG. Menstrual ciclicity after metformin therapy in polycystic ovary syndrome. Obstet Gynecol 1997;90:392-5.

37. Copinschi G, Wegienka LC, Hane S. Effect of arginine on serum levels of insulin and growth hormone in obese subjects. Metabolism 1967;16:485-91.

38. Bell JP, Donald RA, Espiner LA. Pituitary response to insulininduced hypoglycemia in obese subjects before and after fasting. J Clin Endocrinol Metab 1970;31:546-51.

39. Cordido F, Penalva A, Dieguez C. Massive growth hormone $(\mathrm{GH})$ discharge in obese subjects after the combined administration of GH-releasing hormone and GHRP-6: evidence for a marked somatotroph secretory capability in obesity. J Clin Endocrinol Metab 1993;96:819-23.

40. Dieguez C, Carro E, Seoane L. Regulation of somatotroph cell function by the adipose tissue. Int J Obes 2000;24:S100-3.

41. Golay A, Swislocki ALM, Chen Y-DI, Jaspan JB, Reaven GM. Effect of obesity on ambient plasma glucose, free fatty acid, insulin, growth hormone and glucagon concentrations. J Clin Endocrinol Metab 1986;63:481-4.

42. Maccario M, Tassone F, Gauna C. Effects of short-term administration of low-dose rhGH on IGF-1 levels in obesity and Cushing's syndrome: indirect evaluation of sensitivity to GH. Eur J Endocrinol 2001;144:251-6.

43. Mancini MC, Costa APAC, Melo ME, Cercato C, GiannellaNeto D, Garrido Jr AB, et al. Effect of gastric bypass on spontaneus growth hormone and ghrelin release profiles. Obes Res 2006; 14:383-7.

44. Glass AR. Endocrine aspects of obesity. Med Clin North Am 1989;73:139-60.

45. Gianotti L, Pivetti S, Lanfranco F, Tassone F, Navone E, Vittori $\mathrm{R}$, et al. Concomitant impairment of growth hormone secretion and peripheral sensitivity in obese patients with obstructive sleep apnea syndrome. J Clin Endocrinol Metab 2002;87(11):5052-7.

46. Shadid S, Jensen MD. Effects of growth hormone administration in human obesity. Obes Res 2003;11(2):170-5.

47. Halpern A, Mancini MC, Cercato C, Villares SMF, Costa APAC. Efeito do hormônio do crescimento sobre parâmetros antropométricos e metabólicos na obesidade andróide. Arq Bras Endocrinol Metab 2006;50(1):68-73.
48. Moore R, Grant AN, Howard AM, Mills IH. Treatment of obesity with triodothyronine and a very-low calorie liquid formula diet. Lancet 1980;223-6.

49. Marcin Krotkiewski. Thyroid hormones in the pathogenesis and treatment of obesity. Eur J Pharmacol 2002;440:85-98.

50. Kralik A, Eder K, Kirchgessner M. Influence of zinc and selenium deficiency on parameters relating to thyroid hormone metabolism. Horm Metab Res 1996;28:223-6.

51. Krotkiewski M, Holm G, Shono N. Small dose of triiodothyronine can change some risk factors associated with abdominal obesity. Int J Obes 1997;21:922-9.

52. Krotkiewski M. Thyroid hormones in the pathogenesis and treatment of obesity. Eur J Pharmacol 2002;440:85-98.

53. Moulin de Moraes CM, Mancini MC, de Melo ME, Figueiredo DA, Villares SM, Rascovski A, et al. Prevalence of subclinical hypothyroidism in a morbidly obese population and improvement after weight loss induced by Roux-en-Y gastric bypass. Obes Surg 2005;15(9):1287-91.

54. Adsani H, Hoffer LJ, Silva JE. Resting energy expenditure is sensitive to small dose changes in patients on chronic thyroid hormone replacement. $J$ Clin Endocrinol Metab 1997;82:1118-25.

55. Caraccio N, Ferrannini E, Monzani F. Lipoprotein profile in subclinical hypothyroidism: response to levothyroxine replacement, a randomized placebo-controlled study. J Clin Endocrinol Metab 2002;87:1533-8.

56. lossa S, Lionetti L, Mollica MP, Crescenzo R, Barletta A, Liverini $\mathrm{G}$. Fat balance and serum leptin concentration in normal, hypothyroid and hyperthyroid rats. Int J Obes $2001 ; 25(3): 417-25$.

Endereço para correspondência:

Roberta A. Lordelo

Rua Teodoro Sampaio 363, apto. 612

05405-000 São Paulo, SP

E-mail: rlordelo21@gmail.com 